\title{
Polymorphism of mycotoxin biosynthetic genes among Fusarium equiseti isolates from Italy and Poland
}

\author{
Lukasz Stępień • Karolina Gromadzka • \\ Jerzy Chelkowski
}

Received: 28 July 2011 / Revised: 10 January 2012 / Accepted: 25 January 2012 /Published online: 22 February 2012

(C) The Author(s) 2012. This article is published with open access at Springerlink.com

\begin{abstract}
Fusarium equiseti (Corda) Saccardo is a soil saprophyte and a weak pathogen, associated with several diseases of fruit and other crops in subtropical and tropical areas, but also in countries with temperate climate. A wide range of secondary metabolites has been identified among natural $F$. equiseti populations, with zearalenone (ZEA), fusarochromanone and fusarenon-X being the most common. In present study, the genetic diversity of strains from two populations (from Italy and Poland) was evaluated by analysing the translation elongation factor $1 \alpha(t e f-1 \alpha)$ sequences, two polyketide synthases from the ZEA biosynthetic pathway (PKS13 and PKS4) and the TRI5 gene from the trichothecene biosynthetic pathway. ZEA was produced in rice cultures by 20 of the 27 tested isolates in concentrations ranging from $1.34 \mathrm{ng} / \mathrm{g}$ to $34,000 \mathrm{ng} / \mathrm{g}$ ). The ability to produce enniatins and trichothecenes was evaluated in all strains by identifying esynl, TRI13 and TRI4 genes. The presence of PKS4 and PKS13 genes was confirmed by polymerase chain reaction (PCR) in only some ZEAproducing isolates. Similarly, the TRI5 gene was found in 14 of the 27 isolates tested. This is likely to have been caused by the divergence of those genes between $F$. equiset and $F$. graminearum (the latter species was used for the primers design) and can be exploited in phylogenetic studies. The analysis of the mycotoxin biosynthetic gene
\end{abstract}

\footnotetext{
Ł. Stępień $(\triangle) \cdot$ J. Chełkowski

Department of Metabolomics, Institute of Plant Genetics, Polish Academy of Sciences,

Strzeszyńska 34,

60-479 Poznań, Poland

e-mail: 1ste@igr.poznan.pl

K. Gromadzka

Department of Chemistry, Poznań University of Life Sciences,

Wojska Polskiego 75,

60-625 Poznań, Poland
}

sequences can be used to differentiate the studied genotypes even more precisely than the analysis of the non-coding regions (like tef-1 $\alpha$ ).

Keywords Biosynthetic genes · DNA polymorphism .

Fusarium $\cdot$ Mycotoxins $\cdot$ Zearalenone $\cdot$ Phylogenetic markers

\section{Introduction}

Fusarium equiseti (Corda) Saccardo is a common soil saprophyte associated with several fruit and other plant tissue diseases of agriculturally important crops. The fungus is typical mostly for subtropical and tropical areas, but is also isolated from soil and plant hosts in countries of temperate climate zone, like Norway, Canada, Russia and Poland (Booth 1971; Kosiak et al. 2005; Marasas et al. 1984). The list of host species includes cereals (wheat, triticale, maize and rice), asparagus, cotton, potato, tomato, cowpea, onion and ginseng (Punja et al. 2008). Besides cultivated plants, it also colonises wild species, including herbal plants and trees, e.g. Equisetum L., Chenopodium L. and Pinus L. Although $F$. equiseti is considered as moderately aggressive, it is capable of synthesising a vast range of toxic secondary metabolites, which exhibit both phyto- and cytotoxicity (Langseth et al. 1998-1999; Morrison et al. 2002). These include the trichothecenes: 4-acetylnivalenol (fusarenon X, FUS-X), nivalenol (NIV), scirpentriol with its acetyl derivatives (MAS and DAS), and numerous other mycotoxins, such as zearalenone (ZEA), beauvericin (BEA), fusarochromanone (FUSCHR), equisetine (EQ) and butenolide (Thrane 1989).

Recently, ZEA became particularly interesting to the researchers because of its oestrogenic properties and the ability to be accumulated at high levels in naturally infected 
cereal grain (Gromadzka et al. 2008). Besides F. equiseti, ZEA is produced by other Fusarium species, including the most important pathogens of maize and wheat, i.e. F. culmorum and $F$. graminearum. Also, glycosylated derivatives of ZEA were identified both in wheat and in maize grain. They can be regarded as potentially hazardous for human and animal health, as they can be converted to ZEA in the digestive tract (Berthiller et al. 2009; Sulyok et al. 2006). It can be accumulated in cereal grain at high levels, reaching several $\mu \mathrm{g} / \mathrm{g}$, and transferred with infected grain to cereal products, such as animal feed (Gromadzka et al. 2008).

Isolates of $F$. equiseti species are highly variable in a number of morphological traits, including the size of apical cells, conidia shape, aerial mycelium texture, growth rate on standard media, pigmentation and sporodochia appearance (Kosiak et al. 2005). Also, a great diversity has been observed in metabolites composition and amounts among natural F. equiseti populations, but ZEA, FUSCHR and FUS-X are the most commonly synthesised.

To identify and assess the genetic diversity of the natural populations of pathogens, numerous molecular assays have been developed and applied. As far as the Fusarium genus is concerned, analysis of the highly polymorphic intron regions like the translation elongation factor $1 \alpha(t e f-1 \alpha)$ appears as the most useful and versatile tool, as it allows the discrimination of the genotypes even on the sub-specific level (Kristensen et al. 2005; Stępień et al. 2011a).

Therefore, a similar approach was used in this study in order to evaluate the variability of $F$. equiseti isolates from two populations (originating from Italy and Poland, plus an additional strain from Argentina), based on the partial sequences of the $t e f-1 \alpha$. Additionally, we analysed the polymorphism present in the coding regions of the two genes from the ZEA biosynthetic pathway (PKS13 and PKS4) and inside the TRI5 gene, which encodes a key enzyme of the trichothecene biosynthesis pathway-trichodiene synthase. We have examined the level of ZEA biosynthesis in rice cultures and the ability of all isolates to produce enniatins/ beauvericin and trichothecenes (from A and B groups) by the identification of the essential metabolic pathway genes esyn1, TRI13 and TRI4 using gene- and allele-specific polymerase chain reaction (PCR) assays.

\section{Materials and methods}

Fusarium strains and media

Twenty-seven Fusarium strains were included in the study. Twenty-five of them represented two distinct populations of F. equiseti (originating from Italy and Poland). One strain originating from Argentina (ITEM 4323) was also analysed as a representative genotype from a distinct population.
Additionally, one $F$. langsethiae strain (KF 2640) was included in the study (Table 1). For the sequence analyses of TRI5 and PKS13 genes diversity, two strains of F. graminearum (KF 371 representing the nivalenol chemotype and KF 1413 representing the deoxynivalenol chemotype) were used as the outgroup. All isolates were identified using dedicated species-specific PCR-based markers (Table 2). Also, partial sequences of the tef- $1 \alpha$ were amplified for molecular species identification and phylogenetic analysis. The providers of all strains were two culture collections: the KF Fusarium strain collection at the Institute of Plant Genetics, Polish Academy of Sciences, Poznań, Poland, and the ITEM collection at the Institute of Sciences of Food Production (ISPA-CNR), Bari, Italy.

\section{Growth rates}

The strains were examined for growth speed by plating on potato dextrose agar (PDA) medium. The growth speed of 25 isolates described as $F$. equiseti was measured as the colony diameter on $90-\mathrm{mm}$ Petri plates with solid PDA medium (Oxoid, Basingstoke, Hampshire, UK) after 4 days from inoculation. The cultures were grown in the incubator set at room temperature $\left(20^{\circ} \mathrm{C}\right)$ with a $12-\mathrm{h}$ photoperiod. The average was calculated from two replicates.

DNA extraction, PCR primers, cycling profiles and DNA sequencing

Genomic DNA of all isolates was extracted using a hexadecyltrimethylammonium bromide (CTAB) method described in detail previously (Stępień et al. 2008). All primers used for the PCR-based identification of mycotoxin biosynthetic genes, along with references or accession numbers of sequences used for their design, are presented in Table 2. PCRs were done in 25- $\mu$ l aliquots using PTC-200 and C1000 thermal cyclers (Bio-Rad, Hercules, CA, USA). Each reaction tube contained 1 unit of Platinum HotStart Taq DNA polymerase (Invitrogen, Carlsbad, CA, USA), $2.5 \mu \mathrm{l}$ of $10 \times$ PCR buffer, $12.5 \mathrm{pmol}$ of forward/ reverse primers, $2.5 \mathrm{mM}$ of each $\mathrm{dNTP}$ and about 10 $20 \mathrm{ng}$ of fungal DNA. The PCR conditions were as follows: $15 \mathrm{~min}$ at $95^{\circ} \mathrm{C}, 35$ cycles of $\left(30-60 \mathrm{~s}\right.$ at $94^{\circ} \mathrm{C}, 30$ $60 \mathrm{~s}$ at $58-63^{\circ} \mathrm{C}, 1-2 \mathrm{~min}$ at $72^{\circ} \mathrm{C}$ ) and $10 \mathrm{~min}$ at $72^{\circ} \mathrm{C}$. Amplicons were electrophoresed in $1.5 \%$ agarose gels (Invitrogen) with ethidium bromide.

For sequence analysis, PCR-amplified DNA fragments were purified with exonuclease I (Epicentre, Madison, WI, USA) and shrimp alkaline phosphatase (Promega, Madison, WI, USA) using the following programme: $30 \mathrm{~min}$ at $37^{\circ} \mathrm{C}$ and $15 \mathrm{~min}$ at $80^{\circ} \mathrm{C}$. Both strands were labelled using the BigDye Terminator 3.1 kit (Applied Biosystems, Foster 
Table 1 Fusarium equiseti isolates used in this study, along with their country of origin, presence of species-specific and TRI5 gene-specific markers, as well as the amplicon sizes (in base pairs) of partial coding sequences of PKS4 and PKS13 genes from the zearalenone (ZEA) biosynthetic cluster

*In base pairs

\begin{tabular}{|c|c|c|c|c|c|c|c|}
\hline Isolate & Host & Origin & $\mathrm{FeF} / \mathrm{R}$ & TRI5 & TRI4 & PKS4* & PKS13* \\
\hline ITEM 4323 & Equisetum arvense & Argentina & + & - & - & - & - \\
\hline ITEM $2753 F$. avenaceum & Allium cepa & Italy & - & - & - & 500 & - \\
\hline ITEM 3190 & Chenopodium & Italy & + & - & - & - & - \\
\hline ITEM 3673 & Oryza sativa & Italy & + & - & - & 300 & - \\
\hline ITEM 3699 & O. sativa & Italy & - & + & - & - & - \\
\hline ITEM 4586 & Pinus root & Italy & + & - & - & - & - \\
\hline ITEM 4743 & Solanum lycopersicum & Italy & + & - & - & - & - \\
\hline ITEM 5104 & Orobanche ramosa & Italy & + & - & - & 750 & - \\
\hline ITEM 6164 & O. ramosa & Italy & - & + & - & - & 700 \\
\hline ITEM 6461 & Triticum $\mathrm{sp}$. & Italy & + & - & - & - & - \\
\hline ITEM 6463 & Triticum sp. & Italy & + & - & - & 400 & 532 \\
\hline ITEM 7502 & Triticum sp. & Italy & + & - & - & - & - \\
\hline KF 2650 & Soil & Italy & - & + & - & $200 / 355$ & 532 \\
\hline KF 2651 & Soil & Italy & - & + & + & 355 & 532 \\
\hline KF 8 & Triticale & Poland & - & + & - & 355 & 532 \\
\hline KF 13 & T. aestivum & Poland & + & - & + & 750 & - \\
\hline KF 24 & Zea mays & Poland & + & + & - & 750 & 532 \\
\hline KF 72 & Triticale & Poland & - & + & + & 355 & 532 \\
\hline KF 89 & T. aestivum & Poland & - & + & - & 355 & 532 \\
\hline KF 1011 & Lycopersicon esculentum & Poland & + & + & - & 750 & 532 \\
\hline KF 1017 & L. esculentum & Poland & - & - & - & 750 & 532 \\
\hline KF 2652 & Soil & Poland & + & + & - & $200 / 700$ & 532 \\
\hline KF 2653 & Soil & Poland & + & - & - & $355 / 750$ & $200 / 532$ \\
\hline KF 2654 & Soil & Poland & + & + & - & $150 / 750$ & 532 \\
\hline KF 2655 & Soil & Poland & + & + & - & $200 / 750$ & 532 \\
\hline KF 2656 & Soil & Poland & - & + & - & 355 & 532 \\
\hline KF $2640 F$. langsethiae & T. aestivum & Poland & - & + & + & - & 700 \\
\hline
\end{tabular}

City, CA, USA) according to Błaszczyk et al. (2005) and precipitated with ethanol. Sequence reading was performed using Applied Biosystems equipment.

Sequence analysis and phylogeny reconstruction

The sequences of the PCR products were compared to the National Center for Biotechnology Information (NCBI) nucleotide collection using MEGABLAST and initially aligned with the ClustalW algorithm. Phylogenetic relationships were reconstructed with the MEGA4 software package (Tamura et al. 2007) using the maximum parsimony approach (closest neighbour interchange heuristics). No gap-containing positions were considered in the phylogeny analysis. All reconstructions were tested by bootstrapping with 10,000 replicates.

\section{Zearalenone analysis}

Rice cultures were prepared from individual Fusarium isolates (Kostecki et al. 1999; Moretti et al. 2008). Long-grain white rice was used $(50 \mathrm{~g}$ per flask with the addition of $12.5 \mathrm{ml}$ of sterile water), left overnight and sterilised by autoclaving the next day. Samples were inoculated with $4 \mathrm{~cm}^{2}$ of 7-day-old mycelium on PDA medium. The average culture humidity was kept at around $30 \%$ and maintained for 14 days. Then, the cultures were dried at room temperature, ground and homogenised for $3 \mathrm{~min}$ with methanol-water $(3: 1, \mathrm{v} / \mathrm{v})$ mixture $(10 \mathrm{ml}$ for $5 \mathrm{~g}$ of rice culture) and, finally, filtered through Whatman no. 4 filter paper (Sydenham et al. 1990).

ZEA content was determined by the high-performance liquid chromatography (HPLC) method according to the method of Visconti and Pascale (1998). The chromatographic system consisted of Waters HPLC 2695 apparatus with a Waters 2475 Multi $\lambda$ Fluorescence Detector and a Waters 2996 Array Detector. The excitation and emission wavelengths were 274 and $440 \mathrm{~nm}$, respectively. The reservedphase C-18 Nova Pak column $(3.9 \times 150 \mathrm{~mm})$ and acetonitrile-water-methanol $(46: 46: 8, \mathrm{v} / \mathrm{v} / \mathrm{v})$ as the mobile phase at a flow rate of $0.5 \mathrm{ml} / \mathrm{min}$ were used. The detection limit of ZEA was $3 \mathrm{ng} / \mathrm{g}$. 
Table 2 Primers used in this study, expected amplicon sizes, references and GenBank accession numbers of the sequences amplified

\begin{tabular}{|c|c|c|c|}
\hline Marker & $5^{\prime}>3^{\prime}$ sequence & $\begin{array}{l}\text { Amplicon } \\
\text { size (bp) }\end{array}$ & $\begin{array}{l}\text { Accession } \\
\text { number/reference }\end{array}$ \\
\hline $\begin{array}{l}\text { FeF1 } \\
\text { FeR1 }\end{array}$ & $\begin{array}{l}\text { CATACCTATACGTTGCCTCG } \\
\text { TTACCAGTAACGAGGTGTATG }\end{array}$ & 389 & Mishra et al. 2003 \\
\hline $\begin{array}{l}\mathrm{FaF} \\
\mathrm{FaR}\end{array}$ & $\begin{array}{l}\text { AGCATTGTCGCCACTCTC } \\
\text { GTTTGGCTCTACCGGGACTG }\end{array}$ & 920 & Doohan et al. 1998 \\
\hline $\begin{array}{l}\text { Flang } \\
\text { Lanspo }\end{array}$ & $\begin{array}{l}\text { CAAAGTTCAGGGCGAAAACT } \\
\text { TACAAGAAGAGCGTGGCGATAT }\end{array}$ & 310 & Wilson et al. 2004 \\
\hline $\begin{array}{l}\text { Ef728M } \\
\text { Tef1R }\end{array}$ & $\begin{array}{l}\text { CATCGAGAAGTTCGAGAAGG } \\
\text { GCCATCCTTGGAGATACCAGC }\end{array}$ & 600 & Multiple \\
\hline $\begin{array}{l}\text { PKS4_F } \\
\text { PKS4_R }\end{array}$ & $\begin{array}{l}\text { AGACGGCGCAACAAGGGCTG } \\
\text { GCAGTTGCCCGTGTCGGACA }\end{array}$ & 355 & AY495638.1 \\
\hline $\begin{array}{l}\text { PKS13_1 } \\
\text { PKS13_2 }\end{array}$ & $\begin{array}{l}\text { CCCAGCCAAGCCCAGTACGC } \\
\text { ACAGCGGCTGACCTGGGTCA }\end{array}$ & 532 & DQ019316.1 \\
\hline $\begin{array}{l}\text { TRI4F1506 } \\
\text { T4EndR2 }\end{array}$ & $\begin{array}{l}\text { CCCCTGGCTACTCTCGAGA } \\
\text { AAGCTTTGAGAACCTTCAC }\end{array}$ & 550 & Nicholson et al. 2004 \\
\hline $\begin{array}{l}\text { TRI5_1 } \\
\text { TRI5_2 }\end{array}$ & $\begin{array}{l}\text { AGCGACTACAGGCTTCCCTC } \\
\text { AAACCATCCAGTTCTCCATCT }\end{array}$ & 545 & EF661664.1 \\
\hline $\begin{array}{l}\text { TRI13DON-F } \\
\text { TRI13DON-R }\end{array}$ & $\begin{array}{l}\text { CATCATGAGACTTGTKRAGTTTGGG } \\
\text { GCTAGATCGATTGTTGCATTGAG }\end{array}$ & 216 & Nicholson et al. 2004 \\
\hline $\begin{array}{l}\text { TRI13NIV-F } \\
\text { TRI13NIV-R }\end{array}$ & $\begin{array}{l}\text { CCAAATCCGAAAACCGCAG } \\
\text { TTGAAAGCTCCAATGTCGTG }\end{array}$ & 308 & Nicholson et al. 2004 \\
\hline $\begin{array}{l}\text { Esyn_1 } \\
\text { Esyn_2 }\end{array}$ & $\begin{array}{l}\text { GCCGTTGGCGAGCTGGTCAT } \\
\text { GCAAAGCACGCGTCAACGCA }\end{array}$ & 997 & Z18755.3 \\
\hline
\end{tabular}

\section{Results}

Species-specific DNA marker detection, tef- $1 \alpha$ sequence analysis and phylogeny reconstruction

Three species-specific DNA markers FeF1/R1, FaF/R and Flang/Lanspo (Table 2) were used to identify the strains included in the study. Of the $25 \mathrm{~F}$. equiseti strains studied, 16 displayed the amplicon of expected size, while nine genotypes did not amplify the fragment (Table 1). In the cases where, based on the molecular data from speciesspecific marker detection, we were not able to confirm the species identification, we still could prove it thanks to the sequence similarities to the tef-1 $\alpha$ sequences of $F$. equiset $i$ available in the GenBank sequence database using BLASTn. The primers Ef728M/Tef-1R were used successfully in many fungal species and they worked fine in our case, amplifying the fragment of tef- $1 \alpha$ gene using template from all isolates except one (ITEM 6164). Among 27 isolates studied, 22 presented the highest similarity to GenBank accessions of $F$. equiseti (90-99\%). The isolate ITEM 2753 appeared to be $F$. avenaceum and KF 2640 was confirmed as $F$. langsethiae. The ITEM 3699 strain was the most similar to $F$. langsethiae and the ITEM 5104 strain to $F$. avenaceum (Fig. 1). To investigate the strain ITEM 6164 in more detail, a tef-1 $\alpha$ gene fragment adjacent to the standard one was amplified and sequenced using an alternative pair of primers. It was dedicated to identifying other fungal genera, but also with some Fusarium genotypes (results not shown). It displayed the highest level of similarity to $F$. sporotrichioides (97\%), F. graminearum (97\%) and F. fujikuroi (97\%). All the obtained tef- $1 \alpha$ sequences (except ITEM 6164) were submitted to the NCBI GenBank and are publicly available under accession numbers JF966231-JF966256.

\section{Growth rate}

Petri plates with PDA medium were used for the analysis of the growth speed of $25 \mathrm{~F}$. equiseti strains used in the study. In the group of Polish strains, only two genotypes measured $70 \mathrm{~mm}$ (KF 2652 and KF 2655), while all the remaining isolates ranged from 40 to $55 \mathrm{~mm}$ (Fig. 2). The majority of Italian strains grew more slowly than the Polish group, forming a colony of about $25-30 \mathrm{~mm}$ in diameter after four days. However, some exceptions from this rule were recorded (ITEM 3699 and ITEM 6164 reached 64 and $75 \mathrm{~mm}$, respectively). Also, two isolates originating from Italy (KF 2650 and KF 2651) presented growth speed on the level of Polish strains rather than of the Italian group (Fig. 2).

\section{Quantification of zearalenone}

ZEA biosynthesis levels varied among all the $F$. equiseti strains analysed (Fig. 3). ZEA was detected in 20 of the 27 tested isolates. ZEA was produced by $F$. equiseti in concentrations ranging between 1.34 and 34,237 ng/g (>34 $\mu \mathrm{g} / \mathrm{g})$. 
Fig. 1 Most parsimonious tree for the isolates studied, created on the basis of the translation elongation factor $1 \alpha(t e f-1 \alpha)$ sequences. The tree was obtained using the maximum parsimony approach and tested by bootstrapping $(10,000$ replicates) with a cut-off value of $50 \%$. Abbreviations used for the origin and host species: IT - Italy, PL - Poland, AR - Argentina, Os - Oryza sativa, $\mathrm{Zm}$ - Zea mays, $\mathrm{Ea}$ - Equisetum arvense, $\mathrm{Ac}-$ Allium cepa, $\mathrm{Ch}-$ Chenopodium, $\mathrm{Pi}$ - Pinus root, $\mathrm{S} 1$ - Solanum lycopersicum, Or - Orobanche ramosa, $\mathrm{Tr}-$ Triticum sp., Le Lycopersicon esculentum, $\mathrm{Tc}$ - triticale, Ta - Triticum aestivum

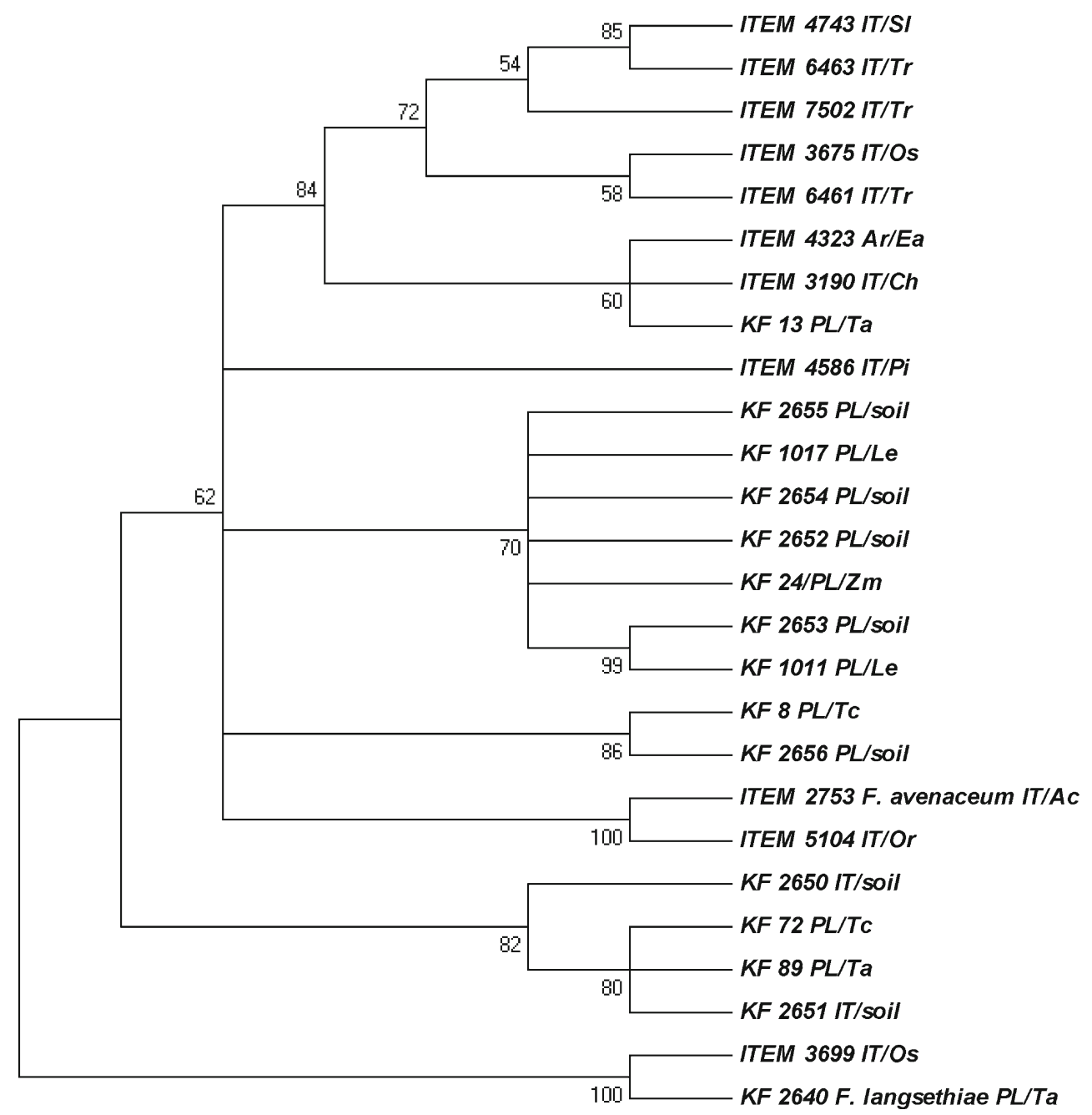

The highest toxin content was observed in Italian isolates: ITEM 5104 and ITEM 3190 (34,237 and 2273.2 ng/g respectively). The other Fusarium isolates were characterised by much lower abilities of the mycotoxin biosynthesis: 14 isolates produced less than $100 \mathrm{ng} / \mathrm{g}$ of the toxin and four isolates' ZEA content only reached the range of 120$460 \mathrm{ng} / \mathrm{g}$.

Sequence polymorphism in two genes from the zearalenone biosynthetic pathway

Based on the sequences of two $F$. graminearum genes encoding polyketide synthases involved in ZEA biosynthesis ( $P K S 4$ and $P K S 13$ ), we designed PCR primers to amplify portions of those genes using genomic DNAs from $F$. equiseti strains. Eight strains from the Italian population of $F$. equiseti did not yield any amplification using the $P K S 4$-specific marker, while among the remaining strains (including $F$. avenaceum ITEM 2753), eight different amplicons were identified (Table 1). Concerning the PKS13 locus, most of the strains displayed the 532-bp allele and two (F. langsethiae KF 2640 and ITEM 6164) amplified a fragment of about $700 \mathrm{bp}$ in length. Eleven strains gave no amplification at all (almost all were from the Italian group). Thus, the PKS4 gene seems to be more polymorphic than PKS13, especially among the isolates from the Polish population. Sequence analysis of the amplified fragments should be performed to reveal the level of this polymorphism, as some of the amplicons can be non-specific. We sequenced several chosen fragments of the PKS13 sequence to confirm the specificity of designed markers and to analyse their intra-specific diversity, as well as similarities between $F$. equiseti and $F$. graminearum. Based on the aligned sequences, a dendrogram was designed (Fig. 4). All the obtained PKS13 sequences were submitted to the NCBI GenBank and are publicly available under accession numbers JF966273-JF966279.

Identification of enniatin and trichothecene biosynthetic genes

To further examine the toxigenic abilities of the strains studied, we used gene-specific DNA markers to prove or exclude the capability of the strains to produce enniatins (by identifying the esyn 1 gene) and trichothecenes (by identifying 
Fig. 2 Colony diameter of the strains used in the study after four days of culturing on potato dextrose agar (PDA) medium at room temperature (mean of two replicates)

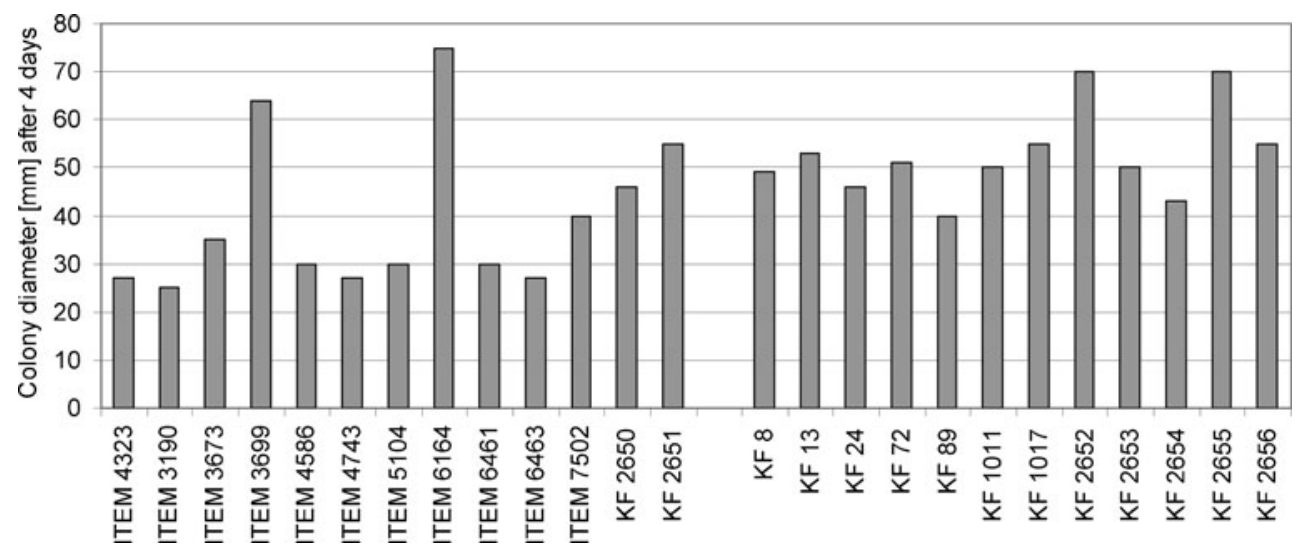

the TRI5 gene and two alleles of the TRI13 gene, associated with deoxynivalenol and nivalenol chemotypes). In case of the esyn 1 gene, encoding enniatin synthetase, we could identify it only in the strain of $F$. avenaceum ITEM 2753. Also, we did not find any amplification products from the strains of $F$. equiseti using primers specific for TRI13DON and TRI13NIV markers, which suggests the inability of those strains to produce DON or NIV. F. graminearum strains KF 1413 and KF 371, exhibiting DON and NIV chemotypes, respectively, amplified the expected amplicons (results not shown).

Since $F$. equiseti has often been reported as a group A trichothecene producer, we screened the studied strain set for the presence of the TRI5 gene and an allele of the TRI4 gene specific for A-trichothecene producers (Nicholson et al. 2004). Only 14 of the strains possessed the TRI5 gene: 13 $F$. equiseti genotypes and KF 2640 F. langsethiae strain (Table 1). The amplified fragments were used to read the sequences, which served as the basis for phylogenetic tree construction (Fig. 5). All the obtained TRI5 sequences were submitted to the NCBI GenBank and are publicly available under accession numbers JF966257-JF966272.

\section{Discussion}

The results of the species-specific DNA marker identification showed that the set of $F$. equiseti isolates used in the study reveals a high level of intra-specific polymorphism. Nine of 25 isolates tested did not amplify the expected amplicon (Table 1). There was no correlation between the country of origin and the presence of the marker fragment. The marker was designed on the basis of the highly variable ITS region of nuclear ribosomal DNA (Mishra et al. 2003), so it seems likely that it displays intra-specific
Fig. 3 Zearalenone (ZEA) production by Fusarium equiseti isolates used in this study

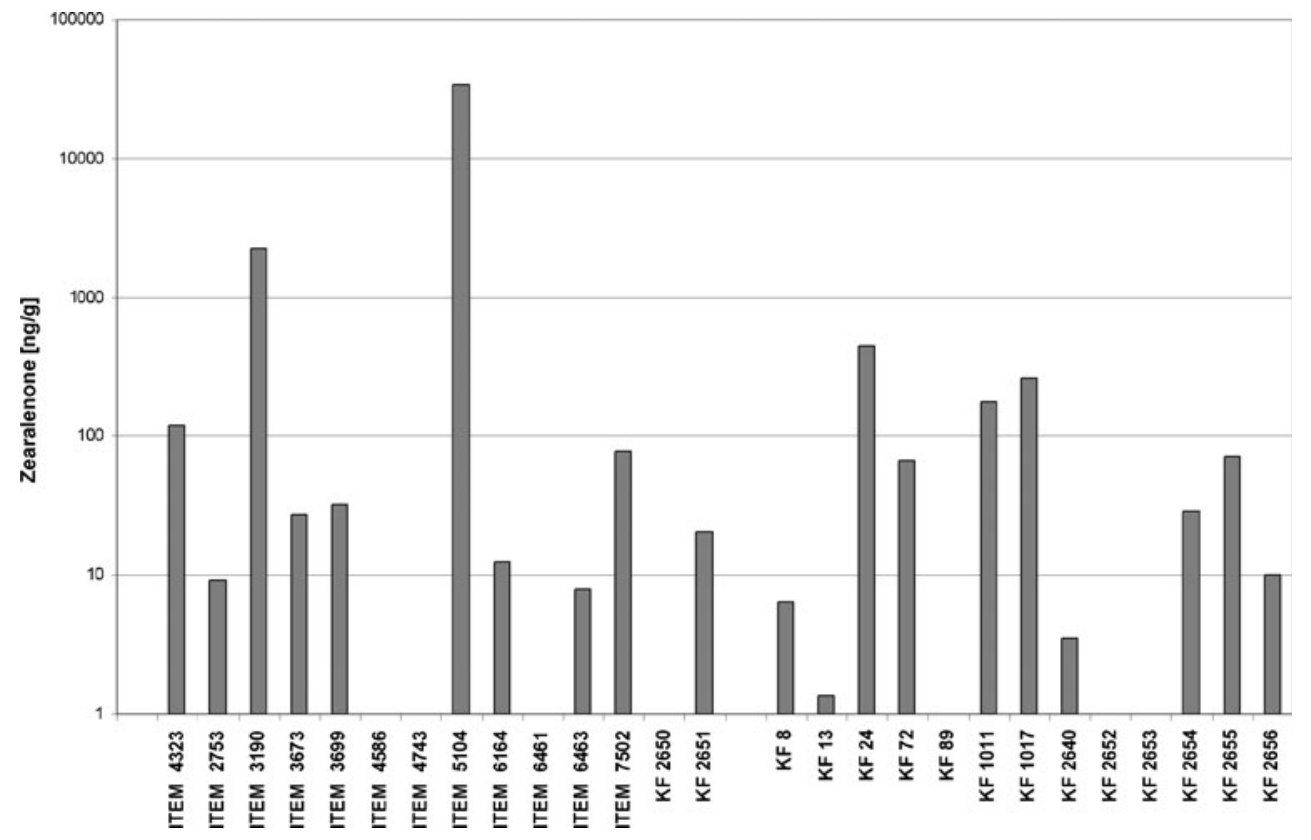




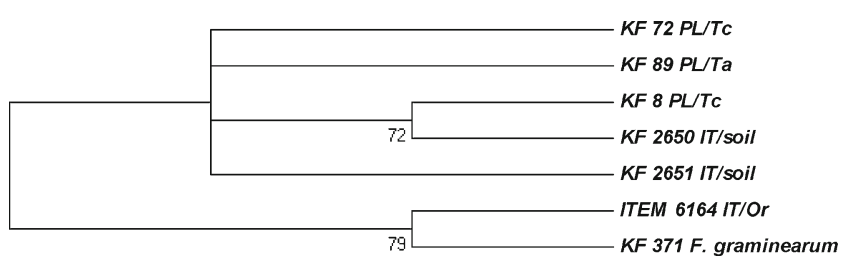

Fig. 4 Most parsimonious tree for six chosen isolates based on the sequences of the polyketide synthase (PKS13) gene from the ZEA metabolic pathway. The tree was obtained using the maximum parsimony approach and tested by bootstrapping (10,000 replicates) with a cut-off value of $70 \%$. The $F$. graminearum KF 371 strain was used as the reference. Abbreviations used for the origin and host species: IT Italy, PL - Poland, Or - Orobanche ramosa, Tc - triticale, Ta Triticum aestivum

polymorphism, which can explain our lack of amplification and identification problems. However, after analysing the tef- $1 \alpha$ sequence, it was proven that eight of nine isolates still represent $F$. equiseti, despite the lack of the amplification of the FeF/FeR marker. Similarly, the analysis of tef- $1 \alpha$ has proven a considerably high level of polymorphism, as it was already postulated before for other members of the Fusarium genus (Kristensen et al. 2005; Stępień et al. 2011b). The only exception was ITEM 6164 strain. We only were able to amplify a fragment of the tef- $1 \alpha$ gene using primers designed for the Clonostachys genus and amplifying a region adjacent to the studied one (results not shown). It is rather unusual concerning the considerable level of conservation present inside this gene (Geiser et al. 2004; Kristensen et al. 2005). The latest findings suggest that sequences which are unique at a species (or group of species) level (e.g. mycotoxin biosynthetic genes) serve as better targets for designing species-specific markers (Alexander et al.
2011; Fernández-Ortuño et al. 2010; Niessen et al. 2004; Proctor et al. 2009; Stępień et al. 2011a).

Based on the obtained tef- $1 \alpha$ sequences, an alignment was prepared and the dendrogram of similarities was calculated (Fig. 1). Concerning only the country of origin, groups of isolates are dispersed, although a group of seven Polish strains from soil, $L$. esculentum and $Z$. mays is distinguishable: $\mathrm{KF}$ 2652, KF 2653, KF 2654, KF 2655, KF 24, KF 1011 and KF 1017. What is even more interesting, a group of four isolates (KF 72, KF 89, KF 2650 and KF 2651) is clearly sharing a rather uncommon variant of the gene, regardless of the origin (two are IT and the other two PL, see Fig. 1). A high level of diversity among $F$. equiseti strains is not surprising, as it was already shown that the tef- $1 \alpha$ gene displays a considerable level of polymorphism among the genotypes of this species (Punja et al. 2008). Also, using different techniques, like restriction fragment length polymorphism (RFLP) and thin layer chromatography (TLC) of the synthesised metabolites, it was possible to differentiate the isolates of common geographical origin (Kosiak et al. 2005). Another remarkable observation is a similarity between the strain ITEM 2753, which was confirmed as $F$. avenaceum, and ITEM 5104. The most likely explanation for this is the misidentification of the strain. However, concerning morphological traits typical for F. equiseti, as well as the presence of the FeF/FeR and PKS4 gene markers (Table 1) and the high level of the in vitro ZEA biosynthesis (Fig. 3), it is also possible that ITEM 5104 strain represents the "off-type" F. equiseti genotype.

As far as growth speed analysis is concerned, the majority of Polish F. equiseti strains grew slightly faster than the Italian ones (Fig. 2). There were two exceptions, however: the strains ITEM 3699 and ITEM 6164 were the fastest growing from the IT population. It seems likely that populations originating
Fig. 5 Most parsimonious tree for the 14 strains tested, based on the trichodiene synthase (TRI5) gene sequences. The maximum parsimony approach was used and tested by bootstrapping $(10,000$ replicates) with a cut-off value of $50 \%$. Two strains of $F$. graminearum (DON chemotype, KF 1413, and NIV chemotype, KF 371) were used as references. Abbreviations used for origin and host species: IT - Italy, PL - Poland, Os - Oryza sativa, $\mathrm{Zm}$ - Zea mays, Or - Orobanche ramosa, Le - Lycopersicon esculentum, $\mathrm{Tc}$ - triticale, Ta - Triticum aestivum

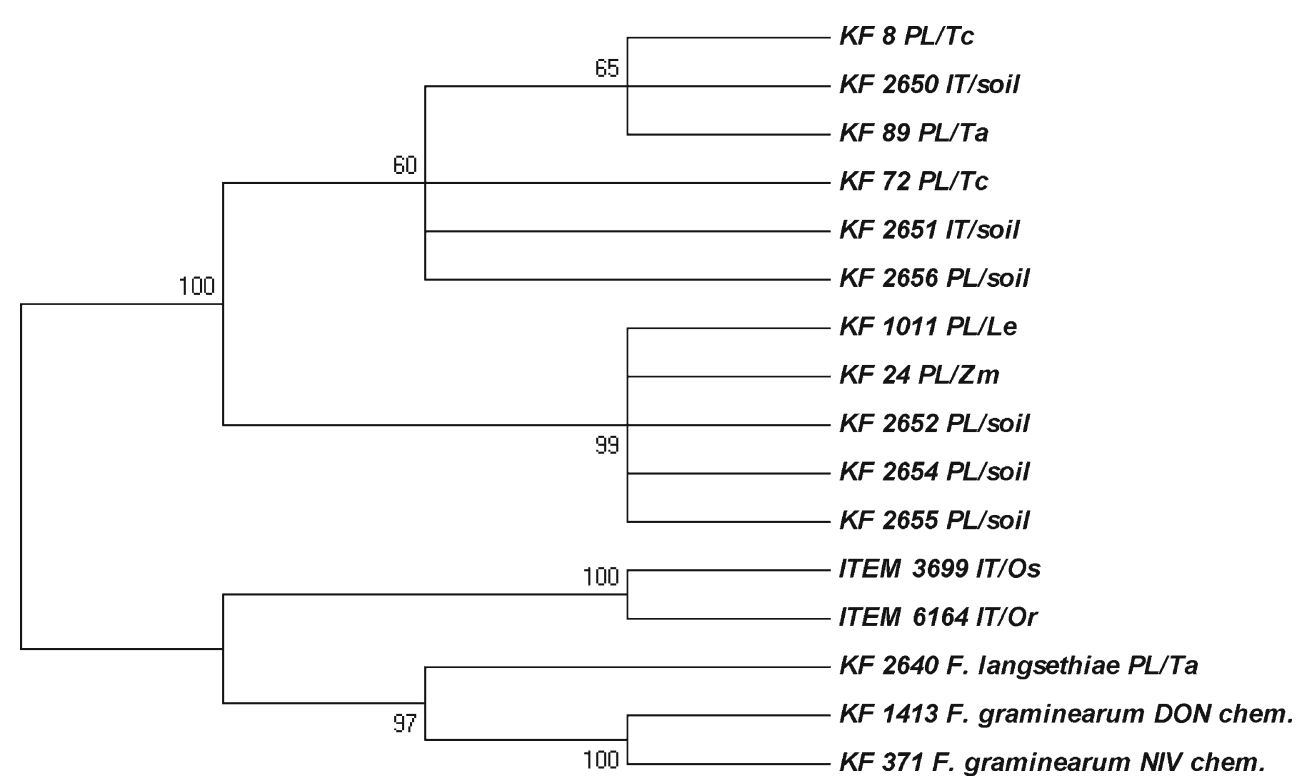


from Southern (i.e. warmer) locations will have an optimum temperature higher than those from temperate climate (PL population). Kosiak et al. (2005) recorded similar colony sizes (39-68 $\mathrm{mm}$ ) for Norwegian isolates as those observed in this study for Polish ones. Canadian isolates from ginseng fields displayed the fastest growth at about $26^{\circ} \mathrm{C}$ (Punja et al. 2008), which seems to be in good agreement with our data. The ITEM 3699 strain was the only one that produced carmine reddish pigment in a PDA culture (results not shown). Having analysed the morphology of conidia and mycelium, and taking the tef- $1 \alpha$ sequence into consideration, it seems likely that the strain represents $F$. langsethiae rather than $F$. equiseti. ITEM 6164 strain's morphology is more typical to $F$. equiseti, but after the strain was subjected to further investigation by analysing the mycotoxin biosynthetic genes TRI5 and PKSI3 (Figs. 4 and 5), it appeared that it probably represents $F$. sporotrichioides.

There are only a few publications on the biosynthesis of ZEA by $F$. equiseti because, until recently, the species was considered as being unable to synthesise ZEA, and its main metabolites were fusarochromanone and trichothecenes (Marasas et al. 1984; Morrison et al. 2002). According to Bosch and Mirocha (1992), ZEA was detected in 8 of the 23 tested isolates of $F$. equiseti at concentrations between 55 and $3,433 \mu \mathrm{g} / \mathrm{g}$. Two of them were lethal to rats, although in culture extracts, the only toxin found was ZEA, at concentrations of 567 and $232 \mu \mathrm{g} / \mathrm{g}$, respectively. According to Langseth et al. (1998-1999), who examined mycotoxin production by $F$. equiseti isolated from Norwegian cereals, three isolates produced ZEA in the range 0.1 to $3 \mu \mathrm{g} / \mathrm{g}$. Kosiak et al. (2005) also found ZEA in all 25 examined isolates of $F$. equiseti, but only 14 of them synthesised this toxin in substantial amounts.

Concerning the relatively high level of ZEA biosynthesis by IT strains (Fig. 3), it seems unexpected that most of them did not amplify PKS4 and PKS13 genes (Table 1). On the contrary, Polish isolates, which generally produced less toxin, showed the presence of both genes, with only one exception (KF 13 did not amplify the PKS13 gene). Obviously, all strains that produce ZEA are supposed to carry $P K S$ genes, despite the negative PCR results. This inconsistency may be explained by the fact that the primers were designed on the basis of $F$. graminearum gene sequences, so a significant level of inter-specific divergence is possible. Specific markers for the reliable identification of $P K S$ genes from $F$. equiseti will be created for future research on the basis of the sequences obtained. Interestingly, similar differentiation of both populations can be visible in the case of TRI5 gene identification. Almost all Polish isolates displayed the presence of the gene, while the Italian isolates did not (Table 1). Again, in the case of the PKS13 sequence, the ITEM 6164 strain appeared as being different from the other strains of $F$. equiseti, grouping with $F$. graminearum (Fig. 4) with a similarity level exceeding $90 \%$.
The abilities of all strains to produce other toxins, such as enniatins and trichothecenes, were analysed by identifying the genes involved in two metabolic pathways. Those were: enniatin synthase gene esyn 1, trichodiene synthase gene TRI5, together with TRI4 marker specific for group A trichothecene producers and TRI13-based markers distinguishing deoxynivalenol and nivalenol chemotypes among trichothecene producers (Nicholson et al. 2004). The esyn 1 gene was present only in the ITEM 2753 strain of $F$. avenaceum, which is the species considered as one of the major producers of this mycotoxin group (Jestoi et al. 2004; Langseth et al. 1998-1999; Logrieco et al. 2002). However, since F. equiseti often produces beauvericin and both cyclohexadepsipeptides (beauvericin and enniatins) share a common biosynthetic pathway, there is a strong possibility that the species possess some form of the synthetase encoded by the esyn 1 gene homologue. Experimental work to prove its presence in F. equiseti using newly designed primers is currently being carried out. Also, both markers amplifying portions of the TRI13 gene gave no amplification from all the strains tested, which should be regarded as the inability to produce DON or NIV by F. equiseti. Actually, the lack of DON/NIV chemotype markers suggests the ability to synthesise group A trichothecenes, like scirpentriol, MAS or DAS, which are, in fact, often produced by this species (Kosiak et al. 2005). TRI4 marker was found in only three strains of $F$. equiseti and one $F$. langsethiae strain (Table 1). This is likely a result of interspecific sequence divergence between $F$. graminearum and $F$. equiseti; however, the marker, despite being described as "generic", has not yet been tested on $F$. equiseti isolates to prove its reliability (Nicholson et al. 2004). Weak amplification fragments obtained in the present work will serve as templates to design species-specific TRI4 markers.

We found the TRI5 gene present in almost all strains from the Polish population and only in a few isolates originating from Italy (Table 1). Two of them (KF 2650 and 2651), though described as Italian strains, based on the tef- $1 \alpha$ and TRI5 sequence comparisons, are placed firmly inside the clade of Polish isolates (Figs. 1 and 5). Also, the speed of their growth resembles the PL strains rather than the IT ones. When tef- $1 \alpha$ and TRI5 dendrograms were compared, two distinct groups of PL isolates from the tef- $1 \alpha$ tree seem to be more dispersed when the TRI5 sequence is analysed. It can be concluded that the Polish strain set used in our study was less uniform than the Italian set, although more isolates should be added to the analyses we have presented here in order to support this hypothesis. Two other strains (ITEM 3699 and ITEM 6164) were already highlighted on the basis of the partly controversial results of the sequence analysis (Figs. 1 and 4). They formed a separate branch on the TRI5 tree, being more closely related to F. langsethiae (KF 2640) and $F$. graminearum variants (KF 1413 and KF 371), as well as to the F. sporotrichioides sequences of TRI5 present in 
GenBank (results not shown), than to those present in the isolates from the Polish population of F. equiseti (Fig. 5).

All our results provide quite a novel insight into the intraspecific polymorphisms of the mycotoxin biosynthetic gene sequences in $F$. equiseti. It shows the divergence of those genes between the species but considerably higher conservation level inside the species. A general conclusion can be drawn that the coding sequences of mycotoxin biosynthetic genes (like TRI5) are as good target sequences for species and population discrimination as highly variable intron regions of genes widely used in phylogenetic studies (e.g. tef- $1 \alpha$ ) and, in some cases, they even have some advantages over the less diverse ones, like the ITS regions.

Open Access This article is distributed under the terms of the Creative Commons Attribution License which permits any use, distribution, and reproduction in any medium, provided the original author(s) and the source are credited.

\section{References}

Alexander NJ, McCormick SP, Waalwijk C, van der Lee T, Proctor RH (2011) The genetic basis for 3-ADON and 15-ADON trichothecene chemotypes in Fusarium. Fungal Genet Biol 48:485-495

Berthiller F, Dall'asta C, Corradini R, Marchelli R, Sulyok M, Krska R, Adam G, Schuhmacher R (2009) Occurrence of deoxynivalenol and its 3- $\beta$-D-glucoside in wheat and maize. Food Addit Contamin Part A Chem Anal Control Expo Risk Assess 26:507-511

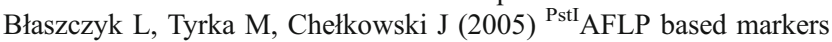
for leaf rust resistance genes in common wheat. J Appl Genet 46:357-364

Booth C (1971) The genus Fusarium. Commonwealth Mycological Institute, Kew, Surrey

Bosch U, Mirocha CJ (1992) Toxin production by Fusarium species from sugar beets and natural occurrence of zearalenone in beets and beet fibers. Appl Environ Microbiol 58:3233-3239

Doohan FM, Parry DW, Jenkinson P, Nicholson P (1998) The use of species-specific PCR-based assays to analyse Fusarium ear blight of wheat. Plant Pathol 47:197-205

Fernández-Ortuño D, Loza-Reyes E, Atkins SL, Fraaije BA (2010) The CYP51C gene, a reliable marker to resolve interspecific phylogenetic relationships within the Fusarium species complex and a novel target for species-specific PCR. Int J Food Microbiol 144:301-309

Geiser DM, del Mar Jiménez-Gasco M, Kang S, Makalowska I, Veeraraghavan N, Ward TJ, Zhang N, Kuldau GA, O'Donnell K (2004) FUSARIUM-ID v. 1.0: a DNA sequence database for identifying Fusarium. Eur J Plant Pathol 110:473-479

Gromadzka K, Waśkiewicz A, Chełkowski J, Goliński P (2008) Zearalenone and its metabolites: occurrence, detection, toxicity and guidelines. World Mycotoxin J 1:209-220

Jestoi M, Rokka M, Yli-Mattila T, Parikka P, Rizzo A, Peltonen K (2004) Presence and concentrations of the Fusarium-related mycotoxins beauvericin, enniatins and moniliformin in Finnish grain samples. Food Addit Contam 21:794-802

Kosiak EB, Holst-Jensen A, Rundberget T, Gonzalez Jaen MT, Torp M (2005) Morphological, chemical and molecular differentation of Fusarium equiseti isolated from Norwegian cereals. Int J Food Microbiol 99:195-206
Kostecki M, Wiśniewska H, Perrone G, Ritieni A, Goliński P, Chełkowski J, Logrieco A (1999) The effects of cereal substrate and temperature on production of beauvericin, moniliformin and fusaproliferin by Fusarium subglutinans ITEM-1434. Food Addit Contam 16:361-365

Kristensen R, Torp M, Kosiak B, Holst-Jensen A (2005) Phylogeny and toxigenic potential is correlated in Fusarium species as revealed by partial translation elongation factor 1 alpha gene sequences. Mycol Res 109:173-186

Langseth W, Bernhoft A, Rundberget T, Kosiak B, Gareis M (1998-1999) Mycotoxin production and cytotoxicity of Fusarium strains isolated from Norwegian cereals. Mycopathologia 144:103-113

Logrieco A, Rizzo A, Ferracane R, Ritieni A (2002) Occurrence of beauvericin and enniatins in wheat affected by Fusarium avenaceum head blight. Appl Environ Microbiol 68:82-85

Marasas WFO, Nelson PE, Toussoun TA (1984) Toxigenic Fusarium species: identity and mycotoxicology. The Pennsylvania State University Press, University Park

Mishra PK, Fox RTV, Culham A (2003) Development of a PCR-based assay for rapid and reliable identification of pathogenic Fusaria. FEMS Microbiol Lett 218:329-332

Moretti A, Mulé G, Ritieni A, Láday M, Stubnya V, Hornok L, Logrieco A (2008) Cryptic subspecies and beauvericin production by Fusarium subglutinans from Europe. Int J Food Microbiol 127:312-315

Morrison E, Rundberget T, Kosiak B, Aastveit AH, Bernhoft A (2002) Cytotoxicity of trichothecenes and fusarochromanone produced by Fusarium equiseti strains isolated from Norwegian cereals. Mycopathologia 153:49-56

Nicholson P, Simpson DR, Wilson AH, Chandler E, Thomsett M (2004) Detection and differentiation of trichothecene and enniatin-producing Fusarium species on small-grain cereals. Eur J Plant Pathol 110:503-514

Niessen L, Schmidt H, Vogel RF (2004) The use of tri5 gene sequences for PCR detection and taxonomy of trichothecene-producing species in the Fusarium section Sporotrichiella. Int J Food Microbiol 95:305-319

Proctor RH, McCormick SP, Alexander NJ, Desjardins AE (2009) Evidence that a secondary metabolic biosynthetic gene cluster has grown by gene relocation during evolution of the filamentous fungus Fusarium. Mol Microbiol 74:1128-1142

Punja ZK, Wan A, Rahman M, Goswami RS, Barasubiye T, Seifert KA, Lévesque CA (2008) Growth, population dynamics, and diversity of Fusarium equiseti in ginseng fields. Eur J Plant Pathol 121:173-184

Stępień Ł, Popiel D, Koczyk G, Chełkowski J (2008) Wheat-infecting Fusarium species in Poland-their chemotypes and frequencies revealed by PCR assay. J Appl Genet 49:433-441

Stępień Ł, Koczyk G, Waśkiewicz A (2011a) FUM cluster divergence in fumonisins-producing Fusarium species. Fungal Biol 115:112-123

Stępień Ł, Koczyk G, Waśkiewicz A (2011b) Genetic and phenotypic variation of Fusarium proliferatum isolates from different host species. J Appl Genet 52:487-496

Sulyok M, Berthiller F, Krska R, Schuhmacher R (2006) Development and validation of a liquid chromatography/tandem mass spectrometric method for the determination of 39 mycotoxins in wheat and maize. Rapid Commun Mass Spectrom 20:2649-2659

Sydenham EW, Gelderblom WCA, Thiel PG, Marasas WFO (1990) Evidence for the natural occurrence of fumonisin $\mathrm{B}_{1}$, a mycotoxin produced by Fusarium moniliforme, in corn. J Agric Food Chem 38:285-290

Tamura K, Dudley J, Nei M, Kumar S (2007) MEGA4: Molecular Evolutionary Genetics Analysis (MEGA) software version 4.0. Mol Biol Evol 24:1596-1599 
Thrane U (1989) Fusarium species and their specific profiles of secondary metabolites. In: Chełkowski J (ed) Fusarium - mycotoxins, taxonomy and pathogenicity. Elsevier, Amsterdam, pp. 199226

Visconti A, Pascale M (1998) Determination of zearalenone in corn by means of immunoaffinity clean-up and high-performance liquid chromatography with fluorescence detection. J Chromatogr A 815:133-140

Wilson A, Simpson D, Chandler E, Jennings P, Nicholson P (2004) Development of PCR assays for the detection and differentiation of Fusarium sporotrichioides and Fusarium langsethiae. FEMS Microbiol Lett 233:69-76 